\title{
Solvent- and Catalyst-Free Microwave-Assisted Decarboxylation of Malonic Acid Derivatives
}

\author{
Fanny Araceli Cabrera-Rivera1, Luis Gabriel Hernández-Vázquez¹, Patricia Flores-Sánchez², \\ Maria Durán-Galván², Jaime Escalante1
}

${ }^{1}$ The Center of Chemical Research, UAEM, Cuernavaca, México

${ }^{2}$ SIGNA Civac S. A. de C. V., Jiutepec, México

Email: jaime@uaem.mx

How to cite this paper: Cabrera-Rivera, F.A., Hernández-Vázquez, L.G., FloresSánchez, P., Durán-Galván, M. and Escalante, J. (2017) Solvent- and Catalyst-Free Microwave-Assisted Decarboxylation of Malonic Acid Derivatives. Green and Sustainable Chemistry, 7, 270-280.

https://doi.org/10.4236/gsc.2017.74021

Received: October 8, 2017

Accepted: November 12, 2017

Published: November 15, 2017

Copyright $\odot 2017$ by authors and Scientific Research Publishing Inc. This work is licensed under the Creative Commons Attribution International License (CC BY 4.0).

http://creativecommons.org/licenses/by/4.0/

\begin{abstract}
It has been found that microwave assisted decarboxylation of malonic acid derivatives can be achieved under solvent-free and catalyst free conditions. This new method produces the corresponding carboxylic acid in a pure manner and with a high yield in a very short reaction time: 3 - $10 \mathrm{~min}$. In general terms, the condition under which this reaction is carried out accelerates the decarboxylation significantly of a series of disubstituted malonic acid derivatives, and makes this new process efficient, easy and environmentally friendly.
\end{abstract}

\section{Keywords}

Microwave, Decarboxylation, Malonic Acid Derivatives, Solvent-Free, Catalyst-Free, Green Chemistry

\section{Introduction}

The synthesis of carboxylic acid derivatives via malonic ester alkylation, hydrolysis, and subsequent decarboxylation is a well-established method for carboncarbon bond formation [1] [2] [3], and it is one of the most important reactions in organic synthesis. Each of these steps is under constant revision with the aim of developing more efficient procedures in terms of stereocontrol, use of friendly reaction conditions and better yields [4].

The key step in this multistep reaction sequence is the decarboxylation process, which is usually performed under harsh thermal conditions (up to $200^{\circ} \mathrm{C}$ ) using conventional [5] [6] or microwave heating [1].

In thermal conditions, the decarboxylation step can be carried out using xylene, aniline, dimethylaniline, quinoline, dioxane, pyridine [7], toluidine, xylidine [8], catechol, resorcinol [9] and acid media [10] [11] as solvents at high 
temperature for long periods of time (overnight). The heating time has been reduced to an hour with the use of cumene as a solvent in the presence of catalytic amounts of pyridine [12]. In addition to those reactions a milder copper-catalyzed decarboboxylation of malonic acid derivatives was reported to proceed in acetronitrile at reflux temperature [3].

Previous reports have established the convenience of microwave assistance as an alternative to high temperatures. For example, Giguere and collaborators carried out the decarboxylation of malonic acid derivatives such as hexanoic acid, butanoic acid, etc. in water and MW conditions: $800 \mathrm{~W}, 190^{\circ} \mathrm{C}$ for $15 \mathrm{~min}$ [13]. Also, Helavi and colleagues have used microwave irradiation (medium to low power) to carry out the decarboxylation of malonic acid derivatives using poly4 -vinyl pyridine as a catalyst in DMF for a short time (3 - $4 \mathrm{~min}$ ) [14]. On the other hand, Tellitu and coworkers established a simple solvent-free protocol to promote the decarboxylation step from mono- and di-substituted malonate monoacid derivatives with short periods of irradiation ( $1-4 \mathrm{~min})$ but using stoichiometric quantities of imidazole [4].

The decarboxylation reaction has been extensively studied and milder conditions have been found. For example, malonic acid derivatives undergo decarboxylation when treated with $N, N$-carbonyldiimidazole $(C D I)$ at room temperature to generate the carbonyl imidazole moiety in high yield [1].

In our research group, we have tried to reproduce some of the reported methods to get impurities related to Valproic acid VPA, which is used as an antiepileptic drug and a mood stabilizer [15]-[20]. But even when those methodologies allowed us to get the compounds that we were interested in, they presented drawbacks such as high temperatures for long reaction times, use of catalysts, use of difficult-to-remove solvents, elaborated workup procedures and low yields.

In this work, we report the solvent- and catalyst-free microwave-assisted decarboxylation of malonic acid derivatives; the decarboxylation process takes around $10 \mathrm{~min}$ at $200 \mathrm{~W}, 180^{\circ} \mathrm{C}-190^{\circ} \mathrm{C}$ in an open vessel system. As an additional advantage, this procedure does not require any workup since the required carboxylic acid is the only product obtained.

\section{Result and Discussion}

Scheme 1 shows the malonic acid derivatives prepared from the commercial available diethyl 2-propylmalonate 1 . To synthesize the desired compounds first the malonate 1 was converted into the corresponding enolate using NaHMDS or $t$-BuOK in dry THF and alkylated with the desired alkyl halides to obtain the dialkylated malonates $2 \mathrm{a}-\mathbf{2} \mathbf{e}$ in moderate to excellent yields. Then, the subsequent saponification of dialkylated malonates with potassium hydroxide in ethanolwater mixture [12] resulted in the formation of the malonic acids 3, 3a-3e with moderate to excellent yields.

Table 1 shows the results obtained from the decarboxylation of the compound 3e carried out as an effort to find the best conditions to achieve the decarboxylation 


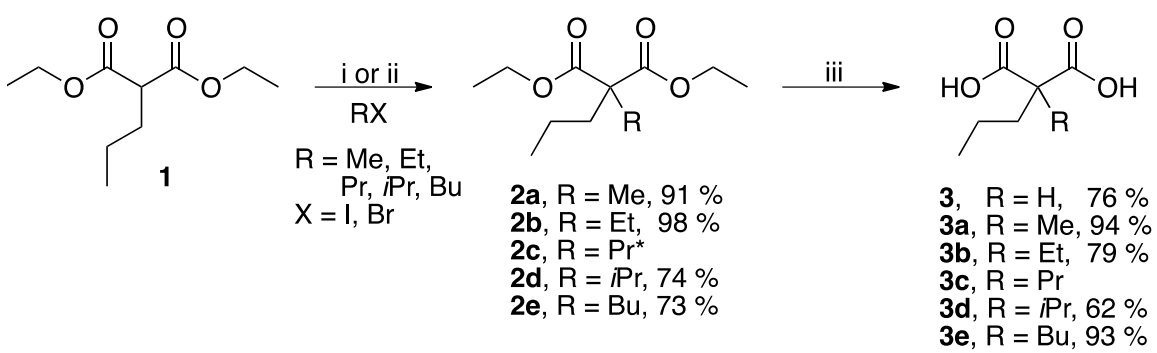

Scheme 1. Synthesis of malonic acid derivatives. Reagents and conditions: i) $t$-BuOK/ THF, RX, rt; ii) NaHMDS/THF, RX, $-15^{\circ} \mathrm{C}$ to rt; iii) $\mathrm{KOH}, \mathrm{H}_{2} \mathrm{O} / \mathrm{EtOH}$, reflux [12]. * Commercially available.

Table 1. Optimization for the decarboxylation of malonic acid 3e.

\begin{tabular}{cccc}
\hline Entry & Conditions & Time $(\mathrm{h})$ & Yield $\mathbf{4 e}(\%)$ \\
\hline 1 & Xylene, reflux & 12 & 11 \\
2 & Py/Xylene. Reflux & 2.0 & 37 \\
3 & Py/Xylene, $\mathrm{MW}$ & 1.5 & 80 \\
& Open Vessel $\left(200 \mathrm{~W}, 180^{\circ} \mathrm{C}-190^{\circ} \mathrm{C}\right)$ & & 98 \\
\hline
\end{tabular}

Each experiment was carried out with $1.48 \mathrm{mmol}$ of 3 e. Experiment 3 was scaled up to $4.2 \mathrm{mmol}$ yielding $60 \%$ of 4 e. Experiment 4 was scaled up to $40 \mathrm{mmol}$ and the yield was also quantitative.<smiles>CCCCC(CCC)(C(=O)O)C(=O)O</smiles>

Scheme 2. Decarboxylation of malonic acid 3e.

of malonic acid derivatives, Scheme 2. Firstly, a common procedure was tested: $3 \mathbf{e}$ was dissolved in xylene and heated to reflux for $12 \mathrm{~h}$ yielding just $11 \%$ of $4 \mathrm{e}$ (entry 1). In a second experiment, $3 \mathbf{e}$ was dissolved again in xylene but this time a catalytic amount of pyridine was added [12]; the mixture was heated to reflux for $2 \mathrm{~h}$ giving $37 \%$ of $4 \mathrm{e}$.

In the chemical synthesis, heat is required mostly to accelerate the reaction rate and is commonly supplied by conduction through warm plate recipients. Microwave (MW) radiation is a different and very efficient alternative to heat the reaction in solution [21] [22]. Microwave-assisted organic synthesis (MAOS) is characterized by the remarkable accelerations produced in many reactions as a consequence or the heating rate, which cannot be reproduced by traditional heating. Higher yields, milder reaction conditions and shorter reaction times can be used and many processes can be improved. Indeed, even reactions that do not occur by conventional heating can be performed using microwaves [23] [24]. Therefore, Microwave (MW) activation as a non-conventional energy source that has become a very popular and useful technology in organic chemistry [25] 
[26]. From experiment 2, it was evident that the presence of a catalytic amount of pyridine was helpful for the decarboxylation by conventional heating and because MAOS has shown a lot of advantages over it. We decided to carry out the same reaction but under microwave irradiation (entry 3 ). In the third experiment, $3 \mathbf{e}$ was dissolved in xylene, a catalytic amount of pyridine was added and the mixture was irradiated at $200 \mathrm{~W}$ and $180^{\circ} \mathrm{C}-190^{\circ} \mathrm{C}$ for $1.5 \mathrm{~h}$ isolating $4 \mathrm{e}$ with $80 \%$ yield. Scaling up experiment 3 was attempted but unfortunately the yield went down.

Microwave assisted chemistry has emerged as a discipline that permeates all aspects of synthetic chemistry. The major goals of this endeavor are to maximize the efficient use of safer raw materials and to reduce waste. On the other hand, Green Chemistry utilizes a set of principles that reduce or eliminate the use or regeneration of hazardous substances in the design, manufacture, and applications of chemical products. One of the key aspects of Green Chemistry is the elimination of solvents in chemical processes or the replacement of hazardous solvents with relatively benign solvents. The development of solvent-free alternative processes is, of course, the best solution [27] [28] [29] [30]. In this field, the microwave heating is proving to be a valuable transformation technique in sustainable chemistry as several methods have been developed using it. Some examples include the use of less solvent compared to the traditional methods and even solvent-free conditions [31]. For this reason, a fourth experiment was carried out in a microwave reactor without solvent and in the absence of catalyst (entry 4); 3e was irradiated at $200 \mathrm{~W}$ and heated up to $180^{\circ} \mathrm{C}-190^{\circ} \mathrm{C}$ and it was found that the reaction finished in just $10 \mathrm{~min}$. The resulting raw material did not need any treatment because $4 \mathbf{e}$ was completely pure; the obtained yield was excellent, $98 \%$.

After the success of experiment 4 , the decarboxylation of malonic acid derivatives $\mathbf{3}, \mathbf{3 a}-\mathbf{3 d}$ was examined under the same conditions. Table 2 summarizes the obtained results.

As we can see in all the experiments the malonic acids derivatives 3, 3a-3d were successfully decarboxylated giving the corresponding carboxylic acid $\mathbf{4}$, $4 a-4 d$ with good to excellent yields $(82 \%-97 \%)$ with very short reaction times ( 3 - $10 \mathrm{~min}$ ). It is important to mention that because the method does not require the use of solvent and catalyst, nor does it need any workup: the products were obtained in a pure manner and no further purification was necessary.

The results show the generality, efficiency, importance and ease of this new method. In this sense, it is necessary to highlight the clearest example of what has just been mentioned and it is the quantitative yield of the decarboxylation process of $3 \mathrm{c}$ to get 2-propylpentanoic acid $\mathbf{4 c}$ (Valproic acid) in just 3 minutes. The decarboxylation step to get VPA usually requires strong bases and high temperatures for at least 2 hours [32]. On the other hand, other groups have described the synthesis VPA in short periods of time using microwave irradiation but in the presence of heterogeneous catalyst and solvents with high boiling point such as DMF [14]. 
<smiles>[R]C(CCC)(C(=O)O)C(=O)O</smiles>

3, $R=H$
3a, $R=M e$
3b, $R=E t$
3c, $R=P r$
3d, R $=\mathbb{P r}$

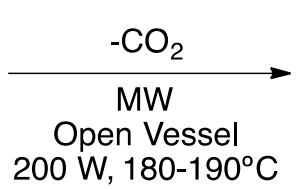<smiles>[R]C(CCC)C(=O)O</smiles>

4, $\mathrm{R}=\mathrm{H}$

4a, $R=M e$

4b, $R=E t$

4c, $\mathrm{R}=\mathrm{Pr}$

4d, $\mathrm{R}=\mathbb{P r}$

Table 2. Solvent- and catalyst-free microwave-assisted decarboxylation of malonic acid derivatives.

\begin{tabular}{cccc}
\hline Entry & Substrate & Time (min) & Yield (\%) \\
\hline 1 & $\mathbf{3}$ & 3 & $\mathbf{4}, 82$ \\
2 & $\mathbf{3 a}$ & 5 & $\mathbf{4 a}, 95$ \\
3 & $\mathbf{3 b}$ & 10 & $\mathbf{4 b}, 91$ \\
4 & $3 \mathbf{c}$ & 3 & $\mathbf{4 c}, 97$ \\
5 & $\mathbf{3 d}$ & 10 & $\mathbf{4 d}, 95$ \\
\hline
\end{tabular}

\section{Experimental}

\subsection{General Information}

The starting materials were obtained from commercial suppliers and were used without purification. The reactions were monitored by thin layer chromatography, on aluminum plates coated with silica gel with fluorescent indicator $\left(60 \mathrm{~F}_{254}\right)$. Microwave irradiation experiments were carried out in Discover CEM equipment. Melting points were measured in open capillary tubes using a Melt-temp Electrothermal apparatus, and are uncorrected. HRMS measurements were obtained on MStation JMS-700 JEOL apparatus. NMR spectra were taken with a Bruker at 300 $\mathrm{MHz}\left({ }^{1} \mathrm{H}\right)$ and $75 \mathrm{MHz}\left({ }^{13} \mathrm{C}\right)$ and Varian Oxford at $400 \mathrm{MHz}\left({ }^{1} \mathrm{H}\right)$ and $100 \mathrm{MHz}$ $\left({ }^{13} \mathrm{C}\right)$ using $\mathrm{CDCl}_{3}$ or $\mathrm{CD}_{3} \mathrm{OD}$ as solvent with TMS as internal standard.

\subsection{General Procedure for the Synthesis of Dialkylmalonates 2a-2e (i)}

1.2 Equiv of $t$-BuOK were suspended in dry THF under nitrogen atmosphere and stirred vigorously at $0^{\circ} \mathrm{C}$. After that malonate 1 ( 1 equiv) was added dropwise and the reaction was stirred for $20 \mathrm{~min}$, then 1.2 equiv of the appropriate alkyl halide was added slowly and the reaction mixture was stirred at $0^{\circ} \mathrm{C}$ for $1 \mathrm{~h}$. Next, the reaction was stirred at room temperature until the malonate 1 disappeared (TLC, hexane:AcOEt 9:1). Upon completion the THF was evaporated under reduced pressure and the residue was suspended in a concentrated solution of $\mathrm{Na}_{2} \mathrm{~S}_{2} \mathrm{O}_{3}$, the product was extracted three times with DCM. The combined organic fractions were dried with anhydrous $\mathrm{Na}_{2} \mathrm{SO}_{4}$ and concentrated. Purification of the raw oily substance by flash column chromatography (hexane:AcOEt, 98:2, 95:5, 90:10) produced the corresponding dialkylmalonate. 


\subsection{General Procedure for the Synthesis of Dialkylmalonates 2a-2e (ii)}

1 Equiv of malonate 1 was dissolved in anhydrous THF under nitrogen atmosphere and the obtained solution was cooled to $-15^{\circ} \mathrm{C}$, followed by dropwise addition of 1.1 equiv of NaHMDS (solution in THF $1 \mathrm{M}$ ). The reaction mixture was stirred for $20 \mathrm{~min}$. Then, 1.2 equiv of the appropriate alkyl halide was added slowly and the reaction mixture was stirred at $-15^{\circ} \mathrm{C}$ for $1 \mathrm{~h}$. Next, the reaction was stirred at room temperature until malonate 1 was consumed (TLC, hexane:AcOEt 9:1). Upon completion, THF was evaporated under reduced pressure and the residue was suspended in a solution of $\mathrm{Na}_{2} \mathrm{~S}_{2} \mathrm{O}_{3}$, the product was extracted three times with DCM. The combined organic fractions were dried with anhydrous $\mathrm{Na}_{2} \mathrm{SO}_{4}$ and concentrated. Purification of the raw oily substance by flash column chromatography (hexane:AcOEt, 98:2, 95:5, 90:10) produced the corresponding dialkylmalonate.

\section{Diethyl 2-methyl-2-propylmalonate 2a}

Colorless oil. ${ }^{1} \mathrm{H} \mathrm{NMR}\left(\mathrm{CDCl}_{3}, 300 \mathrm{MHz}\right): \delta(\mathrm{ppm}) 0.89(\mathrm{t}, J=7.3 \mathrm{~Hz}, 3 \mathrm{H}$, $\left.\mathrm{CH}_{3}\right), 1.21\left(\mathrm{t}, J=7.1 \mathrm{~Hz}, 6 \mathrm{H}, 2 \mathrm{CH}_{3}\right), 1.17-1.27\left(\mathrm{~m}, 2 \mathrm{H}, \mathrm{CH}_{2}\right), 1.36\left(\mathrm{~s}, 3 \mathrm{H}, \mathrm{CH}_{3}\right)$, $1.77-1.82\left(\mathrm{~m}, 2 \mathrm{H}, \mathrm{CH}_{2}\right), 4.14\left(\mathrm{q}, J=7.1 \mathrm{~Hz}, 4 \mathrm{H}, 2 \mathrm{CH}_{2}\right) .{ }^{13} \mathrm{C} \mathrm{NMR}\left(\mathrm{CDCl}_{3}, 75\right.$ $\mathrm{MHz}): \delta(\mathrm{ppm}) 14.2,14.6,17.8,20.0,37.8,53.9,61.2,77.2$, 172.7. HRMS: Calcd for $\mathrm{C}_{11} \mathrm{H}_{20} \mathrm{O}_{4} ; 216.1362$; found: $[\mathrm{M}+\mathrm{H}]^{+} \mathrm{C}_{11} \mathrm{H}_{21} \mathrm{O}_{4}, 217.1497$.

Diethyl 2 -ethyl-2-propylmalonate $2 \mathrm{~b}$

Colorless oil. ${ }^{1} \mathrm{H} \mathrm{NMR}\left(\mathrm{CDCl}_{3}, 300 \mathrm{MHz}\right): \delta(\mathrm{ppm}) 0.81(\mathrm{t}, J=7.6 \mathrm{~Hz}, 3 \mathrm{H}$, $\left.\mathrm{CH}_{3}\right), 0.92\left(\mathrm{t}, J=7.2 \mathrm{~Hz}, 3 \mathrm{H}, \mathrm{CH}_{3}\right), 1.11-1.19\left(\mathrm{~m}, 2 \mathrm{H}, \mathrm{CH}_{2}\right), 1.24(\mathrm{t}, J=7.2 \mathrm{~Hz}$, $\left.6 \mathrm{H}, 2 \mathrm{CH}_{3}\right), 1.81-1.87\left(\mathrm{~m}, 2 \mathrm{H}, \mathrm{CH}_{2}\right), 1.92\left(\mathrm{q}, J=7.6 \mathrm{~Hz}, 2 \mathrm{H}, \mathrm{CH}_{2}\right), 4.17(\mathrm{q}, J=$ $\left.7.1 \mathrm{~Hz}, 4 \mathrm{H}, 2 \mathrm{CH}_{2}\right) .{ }^{13} \mathrm{C} \mathrm{NMR}\left(\mathrm{CDCl}_{3}, 75 \mathrm{MHz}\right): \delta(\mathrm{ppm}) 8.4,14.1,14.4,17.3$, 25.2, 33.9, 57.9, 60.9, 171.9. HRMS: Calcd for $\mathrm{C}_{12} \mathrm{H}_{22} \mathrm{O}_{4}$; 230.1518; found: $[\mathrm{M}+$ $\mathrm{H}]^{+} \mathrm{C}_{12} \mathrm{H}_{23} \mathrm{O}_{4}, 231.1582$.

\section{Diethyl 2-isopropyl-2-propylmalonate $2 \mathrm{~d}$}

Colorless oil. ${ }^{1} \mathrm{H} \mathrm{NMR}\left(\mathrm{CDCl}_{3}, 300 \mathrm{MHz}\right): \delta(\mathrm{ppm}) 0.91\left(\mathrm{t}, J=7.3 \mathrm{~Hz}, 3 \mathrm{H}, \mathrm{CH}_{3}\right)$, $0.97\left(\mathrm{~d}, J=6.9 \mathrm{~Hz}, 6 \mathrm{H}, \mathrm{CH}_{3}\right), 1.16-1.27\left(\mathrm{~m}, 8 \mathrm{H}, \mathrm{CH}_{2}, 2 \mathrm{CH}_{3}\right), 1.82-1.87(\mathrm{~m}, 4 \mathrm{H}$, $2 \mathrm{CH}_{2}$ ), 4.17 (sep, $\left.J=6.9 \mathrm{~Hz}, 1 \mathrm{H}, \mathrm{CH}\right), 4.18\left(\mathrm{q}, J=7.1 \mathrm{~Hz}, 4 \mathrm{H}, 2 \mathrm{CH}_{2}\right) .{ }^{13} \mathrm{C} \mathrm{NMR}$ $\left(\mathrm{CD}_{3} \mathrm{OD}, 75 \mathrm{MHz}\right): \delta$ (ppm) 14.4, 14.7, 18.2, 18.8, 32.0, 36.2, 60.8, 61.9, 171.5.

\section{Diethyl 2-butyl-2-propylmalonate $2 \mathrm{e}$}

Colorless oil. ${ }^{1} \mathrm{H}$ NMR $\left(\mathrm{CDCl}_{3}, 400 \mathrm{MHz}\right): \delta(\mathrm{ppm}) 0.87-0.94\left(\mathrm{~m}, 6 \mathrm{H}, \mathrm{CH}_{3}\right)$, $1.24-1.36\left(\mathrm{~m}, 12 \mathrm{H}, 3 \mathrm{CH}_{2}, 2 \mathrm{CH}_{3}\right), 1.82-1.89\left(\mathrm{~m}, 4 \mathrm{H}, 2 \mathrm{CH}_{2}\right), 4.17$ (q, J=7.1 Hz, $\left.4 \mathrm{H}, 2 \mathrm{CH}_{2}\right) .{ }^{13} \mathrm{C} \mathrm{NMR}\left(\mathrm{CDCl}_{3}, 100 \mathrm{MHz}\right): \delta(\mathrm{ppm}) 14.0,14.4,14.6,17.5,23.1$, 26.3, 32.0, 34.5, 57.7, 61.0, 172.2. HRMS: Calcd for $\mathrm{C}_{14} \mathrm{H}_{26} \mathrm{O}_{4}$; 258.1831; found: $[\mathrm{M}+\mathrm{H}]^{+} \mathrm{C}_{14} \mathrm{H}_{26} \mathrm{O}_{4}, 259.1893$.

\section{2-Propylmalonic acid 3}

White solid; $\mathrm{mp} 88^{\circ} \mathrm{C}-90^{\circ} \mathrm{C} .{ }^{1} \mathrm{H} \mathrm{NMR}\left(\mathrm{CD}_{3} \mathrm{OD}, 500 \mathrm{MHz}\right): \delta(\mathrm{ppm}) 0.79(\mathrm{t}, J$ $\left.=7.4 \mathrm{~Hz}, 3 \mathrm{H}, \mathrm{CH}_{3}\right), 1.22\left(\mathrm{sex}, J=7.5 \mathrm{~Hz}, 2 \mathrm{H}, \mathrm{CH}_{2}\right), 1.66\left(\mathrm{q}, J=7.7 \mathrm{~Hz}, 2 \mathrm{H}, \mathrm{CH}_{2}\right.$ ), $3.14(\mathrm{t}, J=7.5 \mathrm{~Hz}, 1 \mathrm{H}, \mathrm{CH}) .{ }^{13} \mathrm{C} \mathrm{NMR}\left(\mathrm{CD}_{3} \mathrm{OD}, 125 \mathrm{MHz}\right): \delta(\mathrm{ppm}) 14.2,21.8$, 32.3, 52.9, 173.4. HRMS: Calcd for $\mathrm{C}_{6} \mathrm{H}_{10} \mathrm{O}_{4}$; 146.0579; found: $[\mathrm{M}+\mathrm{H}]^{+} \mathrm{C}_{6} \mathrm{H}_{11} \mathrm{O}_{4}$, 
147.0727.

\section{2-Methyl-2-propylmalonic acid 3a}

White solid; mp $93^{\circ} \mathrm{C}-96^{\circ} \mathrm{C} .{ }^{1} \mathrm{H} \mathrm{NMR}\left(\mathrm{CD}_{3} \mathrm{OD}, 400 \mathrm{MHz}\right): \delta(\mathrm{ppm}) 0.93(\mathrm{t}$, $\left.J=7.4 \mathrm{~Hz}, 3 \mathrm{H}, \mathrm{CH}_{3}\right), 1.24-1.34\left(\mathrm{~m}, 2 \mathrm{H}, \mathrm{CH}_{2}\right), 1.36\left(\mathrm{~s}, 3 \mathrm{H}, \mathrm{CH}_{3}\right), 1.77-1.81(\mathrm{~m}$, $\left.2 \mathrm{H}, \mathrm{CH}_{2}\right) .{ }^{13} \mathrm{C}$ NMR $\left(\mathrm{CD}_{3} \mathrm{OD}, 100 \mathrm{MHz}\right): \delta(\mathrm{ppm}) 14.9,18.9,20.7,54.8,176.3$. HRMS: Calcd for $\mathrm{C}_{7} \mathrm{H}_{12} \mathrm{O}_{4} ; 160.0736$; found: $[\mathrm{M}+\mathrm{H}]^{+} \mathrm{C}_{7} \mathrm{H}_{12} \mathrm{O}_{4}, 161.0811$.

2-Ethyl-2-propylmalonic acid $3 \mathrm{~b}$

White solid; $\mathrm{mp} 90^{\circ} \mathrm{C}-93^{\circ} \mathrm{C} .{ }^{1} \mathrm{H}$ NMR $\left(\mathrm{CD}_{3} \mathrm{OD}, 400 \mathrm{MHz}\right): \delta(\mathrm{ppm}) 0.84(\mathrm{t}, J$ $\left.=7.6 \mathrm{~Hz}, 3 \mathrm{H}, \mathrm{CH}_{3}\right), 0.93\left(\mathrm{t}, J=7.2 \mathrm{~Hz}, 3 \mathrm{H}, \mathrm{CH}_{3}\right), 1.17-1.27\left(\mathrm{~m}, 2 \mathrm{H}, \mathrm{CH}_{2}\right), 1.79-$ $1.83\left(\mathrm{~m}, 2 \mathrm{H}, \mathrm{CH}_{2}\right), 1.89$ (q, $\left.J=7.5 \mathrm{~Hz}, 2 \mathrm{H}, \mathrm{CH}_{2}\right) .{ }^{13} \mathrm{C} \mathrm{NMR}\left(\mathrm{CD}_{3} \mathrm{OD}, 100 \mathrm{MHz}\right)$ : $\delta$ (ppm) 9.1, 14.9, 18.7, 26.9, 35.8, 59.2, 175.9. HRMS: Calcd for $\mathrm{C}_{8} \mathrm{H}_{14} \mathrm{O}_{4}$; 174.0892; found: $[\mathrm{M}+\mathrm{H}]^{+} \mathrm{C}_{8} \mathrm{H}_{14} \mathrm{O}_{4}, 175.0968$.

\section{2,2-Dipropylmalonic acid $3 \mathrm{c}$}

White solid; mp $152^{\circ} \mathrm{C}-154^{\circ} \mathrm{C} .{ }^{1} \mathrm{H}$ NMR $\left(\mathrm{CD}_{3} \mathrm{OD}, 400 \mathrm{MHz}\right): \delta$ (ppm) 0.93 (t, $\left.J=7.2 \mathrm{~Hz}, 6 \mathrm{H}, 2 \mathrm{CH}_{3}\right), 1.17-1.27\left(\mathrm{~m}, 4 \mathrm{H}, 2 \mathrm{CH}_{2}\right), 1.79-1.84\left(\mathrm{~m}, 4 \mathrm{H}, 2 \mathrm{CH}_{2}\right)$.

${ }^{13} \mathrm{C}$ NMR $\left(\mathrm{CD}_{3} \mathrm{OD}, 100 \mathrm{MHz}\right): \delta(\mathrm{ppm}) 14.9,18.8,36.4$, 58.7, 175.9. HRMS: Calcd for $\mathrm{C}_{9} \mathrm{H}_{16} \mathrm{O}_{4} ; 188.1049$; found: $[\mathrm{M}+\mathrm{H}]^{+} \mathrm{C}_{9} \mathrm{H}_{16} \mathrm{O}_{4}, 189.1122$.

\section{2-Isopropyl-2-propylmalonic acid $3 \mathrm{~d}$}

White solid; $\mathrm{mp} 119^{\circ} \mathrm{C}-121^{\circ} \mathrm{C} .{ }^{1} \mathrm{H}$ NMR $\left(\mathrm{CD}_{3} \mathrm{OD}, 400 \mathrm{MHz}\right): \delta$ (ppm) 0.92 $\left(\mathrm{t}, J=7.2 \mathrm{~Hz}, 3 \mathrm{H}, \mathrm{CH}_{3}\right), 1.00\left(\mathrm{~d}, 6 \mathrm{H}, 2 \mathrm{CH}_{3}\right), 1.23-1.33\left(\mathrm{~m}, 2 \mathrm{H}, \mathrm{CH}_{2}\right) 1.81-1.86$ $\left(\mathrm{m}, 2 \mathrm{H}, \mathrm{CH}_{2}\right), 2.62$ (sep, $\left.J=6.9 \mathrm{~Hz}, 1 \mathrm{H}, \mathrm{CH}\right) .{ }^{13} \mathrm{C} \mathrm{NMR}\left(\mathrm{CD}_{3} \mathrm{OD}, 100 \mathrm{MHz}\right): \delta$ (ppm) 14.9, 19.0, 19.6, 34.1, 37.7, 62.9, 175.7. HRMS: Calcd for $\mathrm{C}_{9} \mathrm{H}_{16} \mathrm{O}_{4}$; 188.1049; found: $[\mathrm{M}+\mathrm{H}]^{+} \mathrm{C}_{9} \mathrm{H}_{17} \mathrm{O}_{4}, 189.1134$.

\section{2-Butyl-2-propylmalonic acid $3 \mathbf{e}$}

White solid; mp $147^{\circ} \mathrm{C}-149^{\circ} \mathrm{C} .{ }^{1} \mathrm{H}$ NMR $\left(\mathrm{CD}_{3} \mathrm{OD}, 400 \mathrm{MHz}\right): \delta$ (ppm) 0.91 $\left(\mathrm{q}, J=7.1 \mathrm{~Hz}, 6 \mathrm{H}, 2 \mathrm{CH}_{3}\right), 1.14-1.25\left(\mathrm{~m}, 4 \mathrm{H}, 2 \mathrm{CH}_{2}\right), 1.32(\mathrm{sex}, J=7.3 \mathrm{~Hz}, 2 \mathrm{H}$, $\left.\mathrm{CH}_{2}\right), 1.79-1.86\left(\mathrm{~m}, 2 \mathrm{H}, \mathrm{CH}_{2}\right) \cdot{ }^{13} \mathrm{C}$ NMR $\left(\mathrm{CD}_{3} \mathrm{OD}, 100 \mathrm{MHz}\right): \delta(\mathrm{ppm})$ 14.4, 14.9, 18.9, 24.2, 27.8, 34.1, 36.7, 58.7, 176.2. HRMS: Calcd for $\mathrm{C}_{10} \mathrm{H}_{18} \mathrm{O}_{4}$; 202.1205; found: $[\mathrm{M}+\mathrm{H}]^{+} \mathrm{C}_{10} \mathrm{H}_{19} \mathrm{O}_{4}, 203.1294$.

\subsection{General Procedure for the Solvent-Free and Catalyst-Free Microwave-Assisted Decarboxylation of Malonic Acid Derivatives 3, 3a-3e}

The corresponding malonic acid derivative was placed in a round-bottomed flask and was irradiated in open system (open vessel) at $200 \mathrm{~W}$ and $180^{\circ} \mathrm{C}$ $190^{\circ} \mathrm{C}$ for $3-10 \mathrm{~min}$ in a CEM Discover microwave reactor. The reaction was monitored by TLC (hexane:AcOEt, 70:30). Further purification was not necessary because the corresponding product was pure.

\section{Pentanoic acid 4 [33].}

Light yellow oil. ${ }^{1} \mathrm{H}$ NMR ( $\left.\mathrm{CDCl}_{3}, 200 \mathrm{MHz}\right): \delta$ (ppm) $0.90(\mathrm{t}, J=7.2 \mathrm{~Hz}, 3 \mathrm{H}$, $\mathrm{CH}_{3}$ ), 1.35 (sex, $J=7.3 \mathrm{~Hz}, 2 \mathrm{H}, \mathrm{CH}_{2}$ ), 1.61 (quint, $J=7.4 \mathrm{~Hz}, 2 \mathrm{H}, \mathrm{CH}_{2}$ ), 2.34 (t, $\left.J=7.4 \mathrm{~Hz}, 2 \mathrm{H}, \mathrm{CH}_{2}\right), 11.09$ (bs, $\left.1 \mathrm{H}, \mathrm{OH}\right) .{ }^{13} \mathrm{C} \mathrm{NMR}\left(\mathrm{CDCl}_{3}, 50 \mathrm{MHz}\right): \delta(\mathrm{ppm})$ $13.9,22.4,26.9,34.0,180.9$.

2-Methylpentanoic acid 4a [34]. Light yellow oil. The ${ }^{1} \mathrm{H}$ and ${ }^{13} \mathrm{C}$ NMR are in 
agreement with those reported in the literature.

\section{2-Ethylpentanoic acid $\mathbf{4 b}$}

Light yellow oil. ${ }^{1} \mathrm{H}$ NMR $\left(\mathrm{CDCl}_{3}, 300 \mathrm{MHz}\right): \delta(\mathrm{ppm})$ 0.89-0.96 (m, 6H, $\left.2 \mathrm{CH}_{3}\right), 1.25-1.79\left(\mathrm{~m}, 6 \mathrm{H}, 3 \mathrm{CH}_{2}\right), 2.25-2.36(\mathrm{~m}, 1 \mathrm{H}, \mathrm{CH}), 11.5(\mathrm{bs}, 1 \mathrm{H}, \mathrm{OH})$. ${ }^{13} \mathrm{C}$ NMR $\left(\mathrm{CDCl}_{3}, 100 \mathrm{MHz}\right): \delta(\mathrm{ppm}) 11.9,14.2,20.8,25.4,34.1,47.1183 .5$. HRMS: Calcd for $\mathrm{C}_{7} \mathrm{H}_{14} \mathrm{O}_{2} ; 130.0994$; found: $[\mathrm{M}+\mathrm{H}]^{+} \mathrm{C}_{7} \mathrm{H}_{15} \mathrm{O}_{2}, 131.1073$.

2-Propylpentanoic acid 4c [35].

Light yellow oil. ${ }^{1} \mathrm{H}$ NMR ( $\left.\mathrm{CDCl}_{3}, 200 \mathrm{MHz}\right): \delta$ (ppm) $0.90(\mathrm{t}, J=7.0 \mathrm{~Hz}, 6 \mathrm{H}$, $\left.2 \mathrm{CH}_{3}\right), 1.21-1.71\left(\mathrm{~m}, 8 \mathrm{H}, 4 \mathrm{CH}_{2}\right), 2.30-2.41(\mathrm{~m}, 1 \mathrm{H}, \mathrm{CH}), 11.4(\mathrm{bs}, 1 \mathrm{H}, \mathrm{OH})$. ${ }^{13} \mathrm{C}$ NMR $\left(\mathrm{CDCl}_{3}, 50 \mathrm{MHz}\right): \delta(\mathrm{ppm}) 14.2,20.8,34.6,45.4,183.7$.

\section{2-Isopropylpentanoic acid $4 \mathrm{~d}$}

Light yellow oil. ${ }^{1} \mathrm{H}$ NMR $\left(\mathrm{CDCl}_{3}, 400 \mathrm{MHz}\right): \delta(\mathrm{ppm}) 0.91(\mathrm{t}, J=7.2 \mathrm{~Hz}, 3 \mathrm{H}$, $\left.\mathrm{CH}_{3}\right), 0.95\left(\mathrm{dd}, J=6.8 \mathrm{~Hz}, J=1.2 \mathrm{~Hz}, 6 \mathrm{H}, 2 \mathrm{CH}_{3}\right), 1.20-1.63\left(\mathrm{~m}, 4 \mathrm{H}, 2 \mathrm{CH}_{2}\right), 1.87$ (oct, $J=6.8 \mathrm{~Hz}, 1 \mathrm{H}, \mathrm{CH}$ ), $2.10-2.15(\mathrm{~m}, 1 \mathrm{H}, \mathrm{CH}), 11.6$ (bs, $1 \mathrm{H}, \mathrm{OH}) .{ }^{13} \mathrm{C}$ NMR $\left(\mathrm{CDCl}_{3}, 100 \mathrm{MHz}\right): \delta$ (ppm) 14.2, 20.3, 20.7, 21.2, 30.7, 31.7, 52.7, 182.8. HRMS: Calcd for $\mathrm{C}_{8} \mathrm{H}_{16} \mathrm{O}_{2} ; 144.115$; found: $[\mathrm{M}+\mathrm{H}]^{+} \mathrm{C}_{8} \mathrm{H}_{16} \mathrm{O}_{2}, 145.1225$.

\section{2-Propylhexanoic acid $4 \mathrm{e}$}

Light yellow oil. ${ }^{1} \mathrm{H}$ NMR ( $\left.\mathrm{CDCl}_{3}, 400 \mathrm{MHz}\right): \delta$ (ppm) $0.89(\mathrm{t}, J=7.3 \mathrm{~Hz}, 6 \mathrm{H}$, $\left.2 \mathrm{CH}_{3}\right), 1.25-1.37\left(\mathrm{~m}, 6 \mathrm{H}, 3 \mathrm{CH}_{2}\right), 1.38-1.48\left(\mathrm{~m}, 2 \mathrm{H}, \mathrm{CH}_{2}\right), 1.55-1.64(\mathrm{~m}, 2 \mathrm{H}$, $\left.\mathrm{CH}_{2}\right), 2.30-2.37(\mathrm{~m}, 1 \mathrm{H}, \mathrm{CH}), 11.4(\mathrm{bs}, 1 \mathrm{H}, \mathrm{OH}) .{ }^{13} \mathrm{C} \mathrm{NMR}\left(\mathrm{CDCl}_{3}, 100 \mathrm{MHz}\right)$ : $\delta(\mathrm{ppm})$ 13.9, 14.0, 20.8, 22.8, 29.7, 32.1, 34.5, 45.6, 183.6. HRMS: Calcd for $\mathrm{C}_{9} \mathrm{H}_{18} \mathrm{O}_{2} ; 158.1307$; found: $[\mathrm{M}+\mathrm{H}]^{+} \mathrm{C}_{9} \mathrm{H}_{18} \mathrm{O}_{2}, 159.1377$.

\section{Conclusions}

In conclusion, the method has shown that the decarboxylation reaction successfully proceeds with brief microwave irradiation in the absence of solvent and without catalyst. The reaction occurs with all of the synthetized malonic acid derivatives in near quantitative yield and no further workup is necessary. These conditions make this process environmentally friendly and hence very attractive for Green Chemistry.

On the other hand, since decarboxylation of malonic acid derivatives is one of the most common reactions in organic synthesis, particularly in a multistep reaction sequence this new and simple method could be useful for preparing a broad variety of biologically active compounds such as $\alpha$ - [36] and $\beta$-amino acids [37], known drugs such as (S)-Dapoxetine [38] [39] one of the more effective and safe drugs for treating premature ejaculation [40] [41] [42] and Tirofiban [43] [44] an antiplatelet drug (antithrombotic).

In particular, our research group is working on microwave-assisted enantioselective decarboxylation.

\section{Acknowledgements}

We would like to thank CONACyT for financial support (Project No. CB2015/ 256653). We are also grateful to SIGNA Civac S. A de C. V. for providing the 
starting compounds.

\section{References}

[1] Lafrance, D., Bowles, P., Leeman, K. and Rafka, R. (2011) Mild Decarboxylative Activation of Malonic Acid Derivatives by 1,10-Carbonyldiimidazole. Organic Letters, 13, 2322-2325. https://doi.org/10.1021/ol200575c

[2] Nakano, K., Ichikawa, Y. and Kotsuki, H. (2011) Efficient and Mild Procedure for the Decarboxylative Cyanomethyl Esterification of Arylmalonic Acids Using $\mathrm{ClCH}_{2} \mathrm{CN} / 1$,8-Diazabicyclo[5.4.0]undec-7-ene. Heterocycles, 83, 2773-2778. https://doi.org/10.3987/COM-11-12363

[3] Baruah, D. and Konwar, D. (2015) Cellulose Supported Copper Nanoparticles as a Versatile and Efficient Catalyst for the Protodecarboxylation and Oxidative Decarboxylation of Aromatic Acids Under Microwave Heating, Catalysis Communications, 69, 68-71. https://doi.org/10.1016/j.catcom.2015.05.029

[4] Tellitu, I., Beitia, I., Díaz, M., Alonso, A., Moreno, I. and Domínguez, E. (2015) An Improved Solvent-Free System for the Microwave-Assisted Decarboxylation of Malonate Derivatives Based on the use of Imidazole. Tetrahedron, 71, 8251-8255. https://doi.org/10.1016/j.tet.2015.09.012

[5] Toussaint, O., Capdevielle, P. and Maumy, M. (1986) The Copper(I)-Catalyzed Decarboxylation of Malonic Acids: A new Mild and Quantitative Method. Synthesis, 12, 1029-1031. https://doi.org/10.1055/s-1986-31861

[6] Kenyon, J. and Ross, W.A. (1951) The Mechanism of the Decarboxylation of Substituted Malonic Acid Derivatives. Journal of the Chemical Society, 3407-3411. https://doi.org/10.1039/jr9510003407

[7] Fraenkel, G., Belford, R.L. and Yankwich, P.E. (1954) Decarboxylation of Malonic Acid in Quinoline and Related Media. Journal of the American Chemical Society, 76, 15-18. https://doi.org/10.1021/ja01630a003

[8] Clark, L.W.J. (1957) The Effect of Toluidines and Xylidine on Malonic Acid. The Journal of Physical Chemistry, 61, 1009-1010. https://doi.org/10.1021/j150553a042

[9] Haleem, M.A. (1970) Kinetics of the Decarboxylation of Malonic Acid in Catechol. Collection of Czechoslovak Chemical Communications, 35, 2854-2857. https://doi.org/10.1135/cccc19702854

[10] Clark, L.W. (1960) The Decarboxylation of Malonic Acid in Acid Media. The Journal of Physical Chemistry, 64, 41-43. https://doi.org/10.1021/j100830a010

[11] Calí, P. and Begtrup, M. (2002) Synthesis of Arylglycines by Reaction of Diethyl $N$-Boc-iminomalonate with Organomagnesium Reagents. Synthesis, 2002, 63-64.

[12] Markowski, T., Drescher, S., Meister, A., Blume, A. and Dobner, B. (2014) Structure-Property Relationships in a Series of Diglycerol Tetraether Model Lipids and their Lyotropic Assemblies: the effect of Branching Topology and Chirality. Organic Biomolecular Chemistry, 30, 3649-3662. https://doi.org/10.1039/c4ob00048j

[13] Zara, C.L., Jin, T. and Ciguere, R.J. (2000) Microwave Heating in Organic Synthesis: Decarboxylation of Malonic Acid Derivatives in Water. Synthetic Communications, 30, 2099-2104. https://doi.org/10.1080/00397910008087388

[14] Helavi, V.B., Solabannavar, S.B., Desai, U.V. and Mane, R.B. (2003) Microwave Assisted Hydrolysis of Meldrum's Acid Derivatives and Decarboxylation of Derived Malonic Acids. Journal of Chemical Research, Synopses, 2003, 174-175. https://doi.org/10.3184/030823403103173390 
[15] Shimshoni, J.A., Bialer, M., Wlodarczyk, B., Finnell, R.H. and Yagen, B. (2007) Potent Anticonvulsant Urea Derivatives of Constitutional Isomers of Valproic Acid. Journal of Medical Chemistry, 50, 6419-6427. https://doi.org/10.1021/jm7009233

[16] Phiel, C.J., Zhang, F., Huang, E.Y., Guenther, M.G., Lazar, M.A. and Klein, P.S. (2001) Histone Deacetylase Is a Direct Target of Valproic Acid, a Potent Anticonvulsant, Mood Stabilizer, and Teratogen. The Journal of Biological Chemistry, 279, 36734-36741. https://doi.org/10.1074/jbc.M101287200

[17] Löscher, W. (2002) Basic Pharmacology of Valproate: A Review after 35 Years of Clinical Use for the Treatment of Epilepsy. CNS Drugs, 16, 669-694.

[18] Chateauvieux, S., Morceau, F., Dicato, M. and Diederich, M. (2010) Molecular and Therapeutic Potential and Toxicity of Valproic Acid. Journal of Biomedicine and Biotechnology, 2010, Article ID: 479364. https://doi.org/10.1155/2010/479364

[19] Hrebackova, J., Hrabeta, J. and Eckschlager, T. (2010) Valproic Acid in the Complex Therapy of Malignant Tumors. Current Drug Targets, 11, 361-379. https://doi.org/10.2174/138945010790711923

[20] Machado Ximenes, J.C., Lima Verde, E.C., Naffah-Mazzacoratti, M. da G. and Barros Viana, G.S. (2012) Valproic Acid, a Drug with Multiple Molecular Targets Related to Its Potential Neuroprotective Action. Neuroscience \& Medicine, 3, 107-123. https://doi.org/10.4236/nm.2012.31016

[21] Gedye, R., Smith, F., Westaway, K., Ali, H., Baldisera, L., Laberge, L. and Rousell, J. (1986) The Use of Microwave Ovens for Rapid Organic Synthesis. Tetrahedron Letters, 27, 279-282. https://doi.org/10.1016/S0040-4039(00)83996-9

[22] Giguere, R.J., Bray, T.L. and Duncan, S.M. (1986) Application of Commercial Microwave Ovens to Organic Synthesis. Tetrahedron Letters, 27, 4945-4948. https://doi.org/10.1016/S0040-4039(00)85103-5

[23] De la Hoz, A., Díaz-Ortiz, A. and Moreno, A. (2005) Microwaves in Organic Synthesis. Thermal and Non-thermal Microwave Effects. Chemical Society Reviews, 34, 164-178. https://doi.org/10.1039/B411438H

[24] Rathi, A.K., Gawande, M.B., Zboril, R. and Varma, R.S. (2015) Microwave-Assisted Synthesis-Catalytic Applications in Aqueous Media. Coordination Chemistry Reviews, 291, 68-94. https://doi.org/10.1016/j.ccr.2015.01.011

[25] Perreux, L. and Loupy, A. (2001) A Tentative Rationalization of Microwave Effects in Organic Synthesis According to the Reaction Medium, and Mechanistic Considerations. Tetrahedron 57, 9199-9223. https://doi.org/10.1016/S0040-4020(01)00905-X

[26] Kappe, C.O. (2004) Controlled Microwave Heating in Modern Organic Synthesis. Angewandte Chemie International Edition, 43, 6250-6284. https://doi.org/10.1002/anie.200400655

[27] Anastas, P.T. and Kirchhoff, M.M. (2002) Origin, Current Status, and Future Challenges of Green Chemistry. Accounts of Chemical Research, 35, 686-694. https://doi.org/10.1021/ar010065m

[28] Polshettiwar, V. and Varma, R.S. (2008) Microwave-Assisted Organic Synthesis and Transformations using Benign Reaction Media. Accounts of Chemical Research, 41, 629-639. https://doi.org/10.1021/ar700238s

[29] Anastas, P. and Eghbali, N. (2010) Green Chemistry: Principles and Practice. Chemical Society Reviews, 39, 301-312. https://doi.org/10.1039/B918763B

[30] Surati, M.A., Jauhari, S. and Desai, K.R. (2012) A Brief Review: Microwave Assisted Organic Reaction. Archives of Applied Science Research, 4, 645-661. 
[31] Leadbeater, N.E. and Torenius, H.M. (2002) A Study of the Ionic Liquid Mediated Microwave Heating of Organic Solvents. The Journal of Organic Chemistry, 67, 3145-3148. https://doi.org/10.1021/jo016297g

[32] Fu, J.-G. (2012) The Synthetic Process Improvement of Sodium Valproate. Shandong Huagong, 41, 3-4.

[33] Shaikh, T.M. and Hong, F.-E. (2011) Iron-Catalyzed Oxidative Cleavage of Olefins and Alkynes to Carboxylic Acids with Aqueous tert-Butyl Hydroperoxide. Advanced Synthesis \& Catalysis, 353, 1491-1496.

https://doi.org/10.1002/adsc.201000899

[34] Couperus, P.A., Clague, A.D.H. and van Dongen, J.P.C.M. (1978) Carbon-13 Chemical Shifts of Some Model Carboxilic Acids and Esters. Organic Magnetic Resonance, 12, 590-597. https://doi.org/10.1002/mrc.1270111203

[35] Palmieri, A., Gabrielli, S. and Ballini, R. (2010) Michael Reaction of Nitroalkanes with $\beta$-Nitroacrylates under a Solid Promoter: advanced Regio- and Diastereoselective Synthesis of Nitro-Funcionalized $\alpha, \beta$-Unsaturade Esters and 1,3-Butadiene-2- Carboxylates. Advanced Synthesis \& Catalysis, 352, 1485-1492.

https://doi.org/10.1002/adsc.201000142

[36] Rogers, L.M.-A., Rouden, J., Lecomte, L. and Lasne, M.C. (2003) Enantioselective Decarboxylation-Reprotonation of an $\alpha$-Amino Malonate Derivative as a Route to Optically Enriched Cyclic $\alpha$-Amino Acid. Tetrahedron Letters, 44, 3047-3050. https://doi.org/10.1016/S0040-4039(03)00557-4

[37] Nejman, M., Sliwinska, A. and Zwierzak, A. (2005) New Access to Racemic $\beta^{3}$-Amino Acids. Tetrahedron, 61, 8536-8541. https://doi.org/10.1016/j.tet.2005.04.077

[38] Torre, O., Gotor-Fernández, V. and Gotor, V. (2006) Lipase-Catalyzed Resolution of Chiral 1,3-Amino Alcohols: Application in the Asymmetric Synthesis of (S)-Dapoxetine. Tetrahedron: Asymmetry, 17, 860-866. https://doi.org/10.1016/j.tetasy.2006.02.022

[39] Tao, Z. and Fanzhi, Z. (2011) Method for Synthesizing Dapoxetine. Patent No. CN 102229538.

[40] Zhu, Y., Liu, Z., Li, H., Ye. D. and Zhou, W. (2015) A Novel and Practical Asymmetric Synthesis of Dapoxetine Hydrochloride. Beilstein Journal of Organic Chemistry, 11, 2641-2645. https://doi.org/10.3762/bjoc.11.283

[41] Sasikumar, M. and Nikalje, M.D. (2012) Simple and Efficient of (S)-Dapoxetine. Synthetic Communications, 42, 3061-3067. https://doi.org/10.1080/00397911.2011.575522

[42] Venkatesan, K. and Srinivasan, K.V. (2008) A Stereoselective Synthesis of (S)-Dapoxetine Starting from Trans-Cinnamyl Alcohol. Arkivok, xvi, 302-310.

[43] Huajun, S., Lu, H., Shangjin, Y. and Chuanzhi, L. (2010) Simple and Green Method for Synthesizing 4-(4-pyridinyl)-1-butanol as Key Intermediate of Tirofiban Hydrochloride. Patent No. CN 101898998.

[44] Siebler, M., Hennerinci, M.G., Schneider, D., von Reutern, G.M. Seitz, R.J., Rother, J., Witte, O.W., Hamann, G., Junghans, U., Villringer, A. and Fiebach, J.B. (2011) Safety of Tirofiban in Acute Ischemic Stroke the SaTIS Trial. Stroke, 42, 2388-2392. https://doi.org/10.1161/STROKEAHA.110.599662 